\title{
Picroside II protects myocardium from ischemia/reperfusion-induced injury through inhibition of the inflammatory response
}

\author{
JIAN-ZHE LI $^{1^{*}}$, MEI-QING XIE ${ }^{2 *}$, DAN MO $^{3}$, XIAO-FANG ZHAO ${ }^{4}$, \\ SHU-YI YU ${ }^{5}$, LI-JUAN LIU ${ }^{6}$, CHENG WU ${ }^{1}$ and YANG YANG ${ }^{1}$
}

\begin{abstract}
Departments of ${ }^{1}$ Pharmacy and ${ }^{2}$ Hepatobiliary, Glandular and Peripheral Vascular Surgery, Ruikang Hospital, Guangxi University of Chinese Medicine, Nanning, Guangxi 530011; ${ }^{3}$ Department of Surgery,

Maternal and Child Health Hospital of The Guangxi Zhuang Autonomous Region, Nanning, Guangxi 530003;

${ }^{4}$ Department of Liver Diseases, Ruikang Hospital, Guangxi University of Chinese Medicine, Nanning, Guangxi 530011; ${ }^{5}$ Advanced Research Center, Central South University, Changsha, Hunan 410078;

${ }^{6}$ Department of Pharmacy, Jiangxi Provincial Cancer Hospital, Nanchang, Jiangxi 330029, P.R. China
\end{abstract}

Received June 5, 2015; Accepted July 19, 2016

DOI: $10.3892 / \mathrm{etm} .2016 .3841$

\begin{abstract}
The inflammatory response is important in the pathogenesis of myocardial ischemia/reperfusion (I/R) injury. Picroside II, the primary active constituent of Picrorhizae, has been reported to protect the myocardium from I/R-induced injury, however, the exact mechanism underlying these protective effects remains unclear. The aim of the present study was to investigate the mechanism underlying the protective effects of picroside II on I/R-induced myocardial injury. Adult male Sprague-Dawley rats underwent $1 \mathrm{~h}$ left coronary artery occlusion followed by $3 \mathrm{~h}$ reperfusion. Picroside II was administered $(10 \mathrm{mg} / \mathrm{kg})$ via the tail vein $30 \mathrm{~min}$ prior to left coronary artery occlusion. The results revealed that pretreatment of picroside II could significantly alleviate I/R-induced myocardial injury concomitantly with a decrease in inflammatory factor production. In addition, picroside II was also able to decrease high mobility group box 1 (HMGB1) expression, and release and downregulate the expression of the receptor for advanced glycation end products (RAGE), toll-like receptor (TLR)-2 and TLR-4. Furthermore, picroside II was able to inhibit nuclear factor- $\kappa \mathrm{B}(\mathrm{NF}-\kappa \mathrm{B})$ activation. The
\end{abstract}

Correspondence to: $\mathrm{Dr}$ Shu-Yi Yu, Advanced Research Center, Central South University, 110 Xiang-Ya Road, Changsha, Hunan 410078, P.R. China

E-mail: shuyi_yu0731@126.com

Dr Li-Juan Liu, Department of Pharmacy, Jiangxi Provincial Cancer Hospital, 519 East Beijing Road, Nanchang, Jiangxi 330029, P.R. China E-mail: lijuan_liu0791@126.com

*Contributed equally

Key words: picroside II, myocardial ischemia/reperfusion injury, inflammation, high mobility group box 1 results indicated that the protective effect of picroside II on I/R-induced myocardial injury was associated, at least partly, with inhibition of the inflammatory response by suppressing the HMGB1-RAGE/TLR-2/TLR-4-NF- $\kappa \mathrm{B}$ signaling pathway.

\section{Introduction}

Myocardial ischemia/reperfusion (I/R) is a pathological condition, which is characterized by an initial restriction of the blood supply to the myocardium followed by the subsequent restoration of perfusion (1). Although reperfusion is the most effective treatment used to rescue ischemic myocardium, reperfusion may paradoxically lead to severe or additional myocardial injury. Myocardial I/R injury is a common problem in clinical practice and may result in serious consequences. The exact pathophysiology mechanism underlying myocardial I/R injury is complicated and has yet to be fully understood. However, numerous studies support that the inflammatory response is important in the pathogenesis of myocardial I/R injury $(2,3)$ due to the inhibition of inflammation significantly attenuating I/R-induced myocardial injury $(4,5)$. There is increasing evidence to suggest that the inflammatory response during myocardial I/R is closely associated with high mobility group box 1 (HMGB1) (6,7).

HMGB1 is a non-chromosomal nuclear protein that may be actively secreted from activated immune cells or passively released from necrotic and apoptotic cells (8). Numerous studies demonstrated that extracellular HMGB1 is a potent pro-inflammatory mediator $(9,10)$, and is important in triggering the inflammatory response during myocardial I/R $(11,12)$. Furthermore, it has been reported that HMGB1 exerts its pro-inflammatory effects via its specific receptors, which mainly include a receptor for advanced glycation end products (RAGE), Toll-like receptor (TLR)-2 and TLR-4 (13). The interaction of HMGB1 and its specific receptors activates nuclear factor $-\kappa B$ $(\mathrm{NF}-\kappa \mathrm{B})$, and ultimately leads to increased expression and 
release of numerous inflammatory factors (14). Thus, the HMGB1-RAGE/TLR-2/TLR-4-NF- $\kappa$ B pathway is an important inflammatory signaling pathway (13).

Picroside II is a primary active constituent of traditional Chinese medicine. Picrorhizae has been extensively used in China to treat numerous diseases, including upper respiratory tract diseases, disorder of the liver, dyspepsia and chronic diarrhea (15). Previously, it has been reported that picroside II has anti-inflammatory properties and exerts beneficial effects in the nervous and urinary systems. In the rat model of middle cerebral artery occlusion and reperfusion, pretreatment with picroside II could significantly improve the neurobehavioral function and inhibit neurocyte apoptosis, which was correlated with a decrease of inflammatory factor production by inhibition of the TLR-4/NF- $\kappa B$ signaling pathway (15). In addition, the study by Wang et al (16) demonstrated that picroside II was able to decrease I/R-induced renal fibrosis in rats by inhibition of long-term inflammation. Recently, it has been reported that picroside II has a protective effect on myocardial I/R injury in rats (17). Furthermore, our previous investigation also demonstrated that picroside II was able to inhibit hypoxia/reoxygenation (H/R)-induced cardiomyocyte apoptosis (18); however, the exact mechanism underlying the cardioprotective effects of picroside II in myocardial I/R injury is not fully understood.

Due to inflammation being important in myocardial I/R injury, picroside II having anti-inflammatory properties, and the HMGB1-RAGE/TLR-2/TLR-4-NF- $\kappa$ B pathway being an important inflammatory signaling pathway, the present study aimed to explore whether the protective effect of picroside II on myocardial I/R injury in rats is associated with suppressing the inflammatory response by inhibition of the HMGB1-RAGE/TLR-2/TLR-4-NF-кB signaling pathway.

\section{Materials and methods}

Animals. Male Sprague-Dawley rats (age, 8 weeks; weight, 250-300 g) were obtained from the Experimental Animal Center of Central South University (Changsha, China). All rats were housed in standard laboratory conditions in an air conditioned room at temperature $24 \pm 1^{\circ} \mathrm{C}$, with a $12 \mathrm{~h}$ light/dark cycle with a relative humidity of 50-60\%. All rats had free access to food and water. The study was performed in accordance with the NIH Guide for the Care and Use of Laboratory Animals and was approved by the Central South University Veterinary Medicine Animal Care and Use Committee. The present study was approved by the ethical committee of Ruikang Hospital Affiliated to Guangxi University of Chinese Medicine (Nanning, China).

Experimental protocols. The rats were randomly divided into 3 groups (n=8 in each group): Group 1, sham-operated control (sham), in which the rats were subjected to surgical manipulation without myocardial ischemia; Group 2, the I/R group, in which the rats were subjected to myocardial ischemia for $1 \mathrm{~h}$ followed by reperfusion for $3 \mathrm{~h}$; and Group 3 , the picroside II $+\mathrm{I} / \mathrm{R}$ group, in which the rats were administered $10 \mathrm{mg} / \mathrm{kg}$ picroside II dissolved in sterile saline via the tail vein $30 \mathrm{~min}$ prior to left coronary artery occlusion. The model of myocardial I/R was established according to a previously reported method by Li et al (19). Briefly, rats were anesthetized by an intraperitoneal injection of $45 \mathrm{mg} / \mathrm{kg}$ sodium pentobarbital (Shanghai West Tang Bio-Tech Co., Ltd., Shanghai, China) and then placed in the supine position. The body temperature was maintained at $37^{\circ} \mathrm{C}$ by an electric heating pad. The trachea was cannulated for artificial ventilation at a rate of 55 breaths/min using a volume-controlled rodent respirator, and the lead-II of the electrocardiogram was monitored with subcutaneous stainless steel electrodes. The chest was opened through a thoracotomy in the left 4 intercostal spaces. After the pericardium was incised, the anterior wall of the ventricle was exposed, a 4/0 silk suture was attached to a small curved needle and was placed around the left coronary artery close to its origin. The complete occlusion of the coronary artery was confirmed by an ST segment elevation in lead-II and from the change of the ventricular color (from red to white). After a 1-h occlusion, the rats underwent 3 -h reperfusion. The reperfusion was verified by the return of color in the ischemic area. Sham rats underwent a similar operation with the exception of the coronary artery ligation.

Reagents. Picroside II (purity $>99 \%$ ) was purchased from the Chinese National Institute for the Control of Pharmaceutical and Biological Products (Beijing, China). 2,3,5-triphenyltetrazolium chloride (TTC) was obtained from Sigma-Aldrich (St. Louis, MO, USA). The creatine kinase (CK) and lactate dehydrogenase (LDH) assay kits were purchased from Nanjing Jiancheng Bioengineering Institute (Nanjing, China). The terminal deoxynucleotidyl transferase-mediated dUTP nick-end labeling (TUNEL) kit was from Roche (Indianapolis, IN, USA). The antibodies targeting HMGB1, RAGE, TLR-2, TLR-4 and $\beta$-actin were purchased from Abcam (Cambridge, UK). TRIzol reagent was a product of Invitrogen (Thermo Fisher Scientific, Inc., Waltham, MA, USA). The First Strand cDNA Synthesis kit was obtained from MBI Fermentas, Inc. (Vilnius, Lithuania) and the ELISA kits of TNF- $\alpha$, IL- 6 , IL-1 $\beta$, intercellular adhesion molecule-1 (ICAM-1), HMGB1 and NF- $\kappa$ B p65 were purchased from Shanghai Jiang Lai Biotechnology Co., Ltd. (Shanghai, China).

\section{Assessment of myocardial injury}

Histological evaluation. The morphological changes of the cardiac tissue were evaluated by hematoxylin and eosin staining. Briefly, following 3-h reperfusion, the rats were sacrificed with an overdose of sodium pentobarbital $(220 \mathrm{mg} / \mathrm{kg}$; i.p. injection) and their hearts were harvested. Following the removal of the fatty tissues, the atria and right ventricle, and the remaining left ventricle was fixed in $4 \%$ paraformaldehyde and prior to embedding in paraffin. The paraffin-embedded tissue was sliced into $5 \mu \mathrm{m}$ thick sections, and the sections were then stained with hematoxylin and eosin according to standard protocol (20). The morphological changes to the cardiac tissue were observed under a light microscope (Leica DM4000B; Leica Microsystems GmbH, Wetzlar, Germany).

$C K$ and LDH activity assay. To assess myocardial injury, the activity of CK and LDH was determined. Briefly, after 3-h reperfusion, the blood samples were collected from the carotid artery, and then centrifuged at $1,000 \times \mathrm{g}$ for $20 \mathrm{~min}$ at $4^{\circ} \mathrm{C}$. The activity of CK and LDH was detected using the commer- 
cially available colorimetric assay kits (Nanjing Jiancheng Bioengineering Institute, Nanjing, China). in accordance with the manufacturer's instructions.

Infarct size. The infarct size was measured by TTC staining. Briefly, after 3-h reperfusion the left coronary artery was occluded again at the original site, and $1 \mathrm{ml}$ Evans blue dye $(2 \%)$ was injected via the femoral vein. The risk area was analyzed using negative staining with Evans blue. Next, the rat was sacrificed with an overdose of sodium pentobarbital (220 mg/kg; i.p. injection) and the entire heart was rapidly excised. Following the removal of the fatty tissues, atria and right ventricle, the remaining left ventricle was frozen at $-80^{\circ} \mathrm{C}$. The frozen left ventricle was cut transversely into 5 sections $(\sim 2 \mathrm{~mm})$ and the risk area was separated from the colored non-ischemic area (blue). Furthermore, the sections were incubated in $1 \% \mathrm{TTC}$ at $37^{\circ} \mathrm{C}$ for $15 \mathrm{~min}$, and the infarcted myocardium was stained white, whereas the viable myocardium was stained red. The infarct and the risk area were analyzed using an image analyzer (version 6.0; Media Cybernetics Inc., Rockville, MD, USA), and the infarct size was expressed as a percentage of the risk area volume (\%, infarct size/risk area).

Apoptosis analysis. Cardiomyocyte apoptosis was analyzed by TUNEL staining. Briefly, at the end of $3 \mathrm{~h}$ reperfusion, the heart was removed and washed in phosphate-buffered saline. The anterior wall tissues of the left ventricle were fixed in $4 \%$ paraformaldehyde and embedded in paraffin. The paraffin-embedded cardiac tissues were then cut with a thickness of $5 \mu \mathrm{m}$, and the sections were then stained using a TUNEL kit following the manufacturer's instructions. The number of TUNEL-positive cardiomyocytes was visualized under a light microscope, and the apoptosis percentage was expressed as a ratio of apoptotic cells to total cardiomyocytes per field.

ELISA. Following reperfusion for $3 \mathrm{~h}$, the blood samples were collected and centrifuged $1,000 \times \mathrm{g}$ for $20 \mathrm{~min}$ at $4^{\circ} \mathrm{C}$, and the serum samples were stored at $-80^{\circ} \mathrm{C}$ until further analysis. The concentrations of TNF- $\alpha$ (cat. no JL13202), IL-6 (cat. no JL15538), IL-1 $\beta$ (cat. no JL15543), ICAM-1 (cat. no JL15530) and HMGB1 (cat. no JL13892) in the serum and NF- $\kappa$ B p65 (cat. no JL10501) in nuclear lysates of the myocardium were detected using commercially available ELISA kits (Shanghai Jiang Lai Biotechnology Co., Ltd., Shanghai, China) in accordance with the manufacturer's instructions.

Reverse transcription-quantitative polymerase chain reaction $(R T-q P C R)$ analysis. A $100 \mathrm{mg}$ myocardial tissue sample was collected from each rat for RNA isolation. A mortar and pestle, along with liquid nitrogen, were used for disruption of samples. Homogenization of each sample was completed by using a syringe and needle. Total RNA was extracted from $100 \mathrm{mg}$ myocardium using TRIzol reagent (Invitrogen; Thermo Fisher Scientific, Inc.), and the concentration was determined by a spectrophotometric absorbance measurement at $260 \mathrm{~nm}$. A $1 \mu \mathrm{g}$ RNA aliquot was converted into cDNA using the M-MLV reverse transcriptase kit (Promega Corporation, Madison, WI, USA), and the cDNA was then used for qPCR. Quantitative analysis of the mRNA expression was performed using the ABI 7300 quantitative PCR system (Thermo Fisher Scientific, Inc.) with the Power SYBR-Green PCR Master Mix kit. PCR primers (synthesized by Sangon Biotech Co., Ltd., Shanghai, China). were as follows: HMGB1 (forward, 5'-ATGGGCAAAGGA GATCCTA-3' and reverse, 5'-ATTCATCATCATCATCTT CT-3'); RAGE (forward, 5'-TCTCAGAAGCCCAAGGAA GAGT-3' and reverse, 5'-CCTAGGTCTGAAGGCCCTGAG T-3'); TLR-2 (forward, 5'-ACGCAGTGAGTGGTGCAAGTA T-3' and reverse, 5'-CTTCTTCAATGGGTTCCAGCAA-3'); TLR-4 (forward, 5'-GGCATCATCTTCATTGTCCTTG and reverse, 5'-AGCATTGTCCTCCCACTCG-3') and $\beta$-actin (forward, 5'-AGGGAAATCGTGCGTGAC-3' and reverse, 5'-CGCTCATTGCCGATAGTG-3'). The PCR amplification profiles consisted of denaturation at $95^{\circ} \mathrm{C}$ for $10 \mathrm{~min}$, followed by 40 cycles of denaturation at $95^{\circ} \mathrm{C}$ for $15 \mathrm{sec}$ and annealing at $60^{\circ} \mathrm{C}$ for $60 \mathrm{sec}$. In addition, the relative expression values were normalized to the expression value of $\beta$-actin using the $2^{-\Delta \Delta \mathrm{Cq}}$ method (21). All amplification reaction for each sample was performed four times.

Western blot analysis. Myocardial proteins were extracted using the Tissue Total Protein Extraction kit (Beyotime Institute of Biotechnology, Jiangsu, China). Nuclear proteins were isolated using a Nuclear Extraction kit (Beyotime Institute of Biotechnology) in accordance with the manufacturer's instructions. The protein concentration was quantified by a Bradford protein assay (Beyotime Institute of Biotechnology). Equal amounts of protein (40 $\mu \mathrm{g}$ from each group) were loaded onto a $10 \%$ sodium dodecyl sulfate-polyacrylamide gel, and then transferred onto a polyvinylidene fluoride membrane. Following blocking for $1 \mathrm{~h}$ with $5 \%$ skimmed milk at room temperature, the membranes were incubated with the primary antibody for HMGB1 (rabbit polyclonal antibody; diluted $1 \mu \mathrm{g} / \mathrm{ml}$ $(1: 1,000)$; cat. no. ab191583), RAGE (rabbit polyclonal antibody; 1:1,000; cat. no. ab37647), TLR-2 (rabbit monoclonal antibody; 1:5,000; cat. no. ab108998), TLR-4 (mouse monoclonal antibody; $1: 5,000$; cat. no. ab30667) and $\beta$-actin (rabbit polyclonal antibody; 1:1,000; cat. no. ab1801) overnight at $4^{\circ} \mathrm{C}$, followed by incubation with the corresponding horseradish-conjugated secondary antibody (goat anti-rabbit; 1:10,000; cat. no. ab175773 or goat anti-mouse; 1:10,000; cat. no. ab97040) for $1 \mathrm{~h}$ at room temperature. The protein bands were quantified using scanning densitometry, and the results were normalized to the expression of $\beta$-actin.

Statistical analysis. SPSS statistical software was used for statistical analysis (version 18.0; SPSS, Inc., Chicago, IL, USA) Data are expressed as the mean \pm standard error of the smean. All the values were analyzed using analysis of variance and the Newman-Keuls Student's t-test. $\mathrm{P}<0.05$ was considered to indicate a statistically significant difference.

\section{Results}

Effect of picroside II on I/R-induced myocardial injury. As shown in Fig. 1A, myocardial infarction was not observed in the sham group. In the I/R group, the infarct size was $52.95 \pm 3.98 \%$ following ischemia for $1 \mathrm{~h}$ and reperfusion for 
A

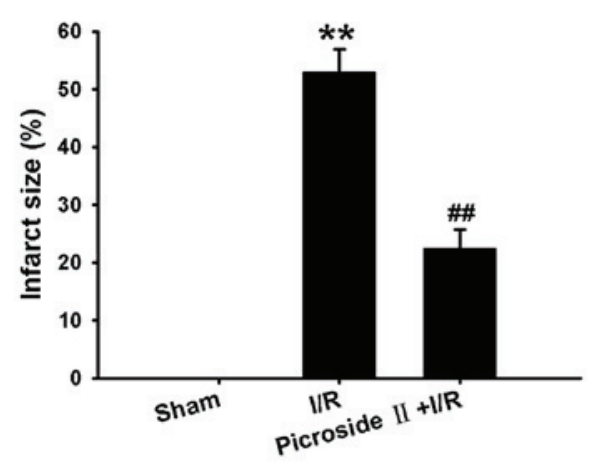

C
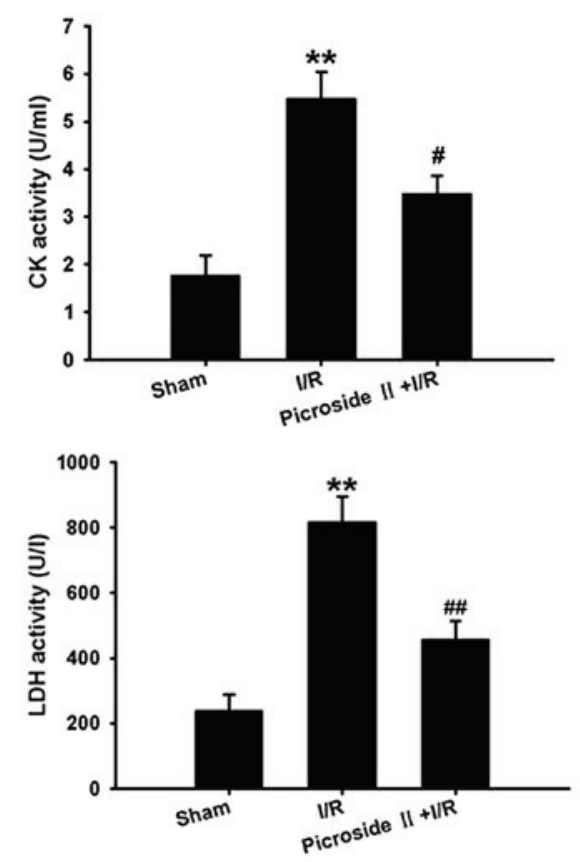

B

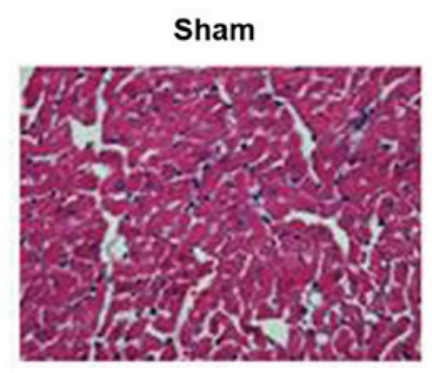

I/R

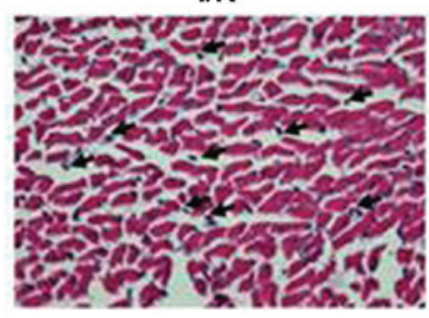

Picroside II+I/P

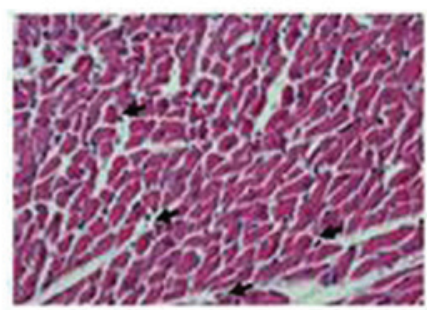

Figure 1. Effect of picroside II on I/R-induced myocardial injury in rats ( $\mathrm{n}=8$ in each group). (A) Infarct size (expressed as the percentage of the area at risk). (B) Representative image of hematoxylin and eosin staining. (C) CK and LDH activity. ${ }^{* *} \mathrm{P}<0.01$, vs. Sham; ${ }^{*} \mathrm{P}<0.05$ and ${ }^{\# \#} \mathrm{P}<0.01$, vs. I/R. I/R, ischemia/reperfusion; CK, creatine kinase; LDH, lactate dehydrogenase.

$3 \mathrm{~h}$. Hematoxylin and eosin staining revealed that the structure of the myocardium in the sham group was arranged regularly, and the cell boundary was clear. Compared with the sham group, the myocardial structure in the I/R group presented irregularity, with some of cells shrinking and getting distorted or ruptured. There was marked inflammatory cell infiltration in the myocardial intercellular space (indicated by the arrows; Fig. 1B). Consistent with the above results, the activity of CK and LDH that were used as indicators of myocardial injury were markedly increased following ischemia for $1 \mathrm{~h}$ and reperfusion for $3 \mathrm{~h}$ (Fig. 1C). However, pretreatment of picroside II could markedly inhibit these effects induced by myocardial I/R (Fig. 1).

Effect of picroside II on I/R-induced cardiomyocyte apoptosis. TUNEL staining revealed that the percentage of apoptotic cells was remarkably increased following ischemia for $1 \mathrm{~h}$ and reperfusion for $3 \mathrm{~h}$ compared with the sham group. However, this effect induced by I/R was significantly ameliorated by administration of picroside II (Fig. 2).
Effect of picroside II on I/R-induced inflammatory factor production. Following ischemia for $1 \mathrm{~h}$ and reperfusion for $3 \mathrm{~h}$, the concentration of TNF- $\alpha$, IL- 6 , IL- $1 \beta$ and ICAM- 1 were remarkably increased compared with the sham group. However, this effect induced by I/R was significantly attenuated by pretreatment of picroside II (Fig. 3).

Effect of picroside II on I/R-induced HMGBI expression and release. qPCR and western blotting analysis demonstrated that the HMGB1 mRNA and protein expression were significantly upregulated in the I/R group compared with that in the sham group. Consistent with these results, the concentration of HMGB1 in the serum was also significantly increased in the $\mathrm{I} / \mathrm{R}$ group. However, the increased expression and concentration of HMGB1 were significantly attenuated by pretreatment with picroside II (Fig. 4).

Effect of picroside II on I/R-induced HMGBI receptor expression. qPCR analysis demonstrated that the mRNA expression of RAGE, TLR-2 and TLR-4 was markedly upregulated after isch- 
A

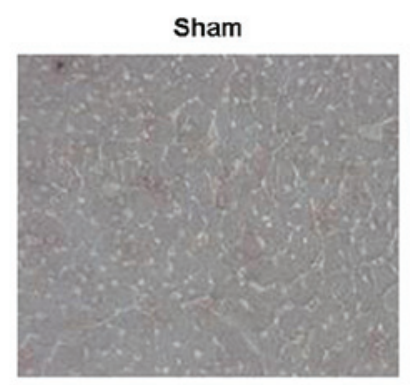

I/R

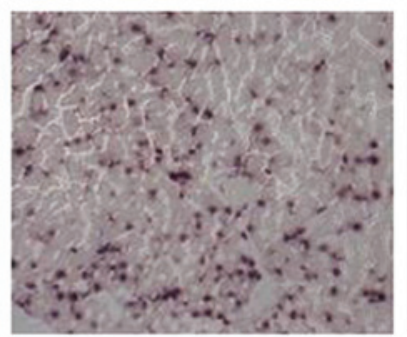

Picroside II + //R

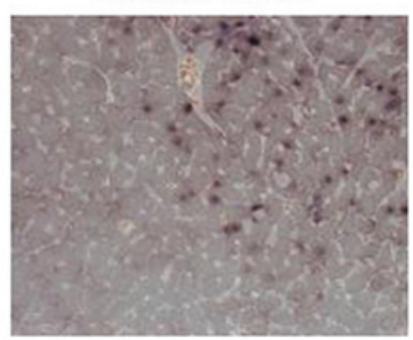

B

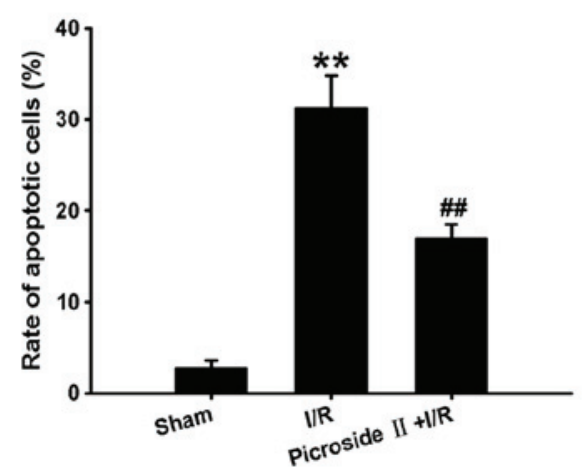

Figure 2. Effect of picroside II on I/R-induced cardiomyocyte apoptosis in rats ( $\mathrm{n}=8$ in each group). (A) Representative image of terminal deoxynucleotidyl transferase-mediated dUTP nick-end labeling staining. (B) Rate of apoptotic cells. ${ }^{* *} \mathrm{P}<0.01$, vs. Sham, and ${ }^{\# \#} \mathrm{P}<0.01$, vs. I/R. I/R, ischemia/reperfusion.
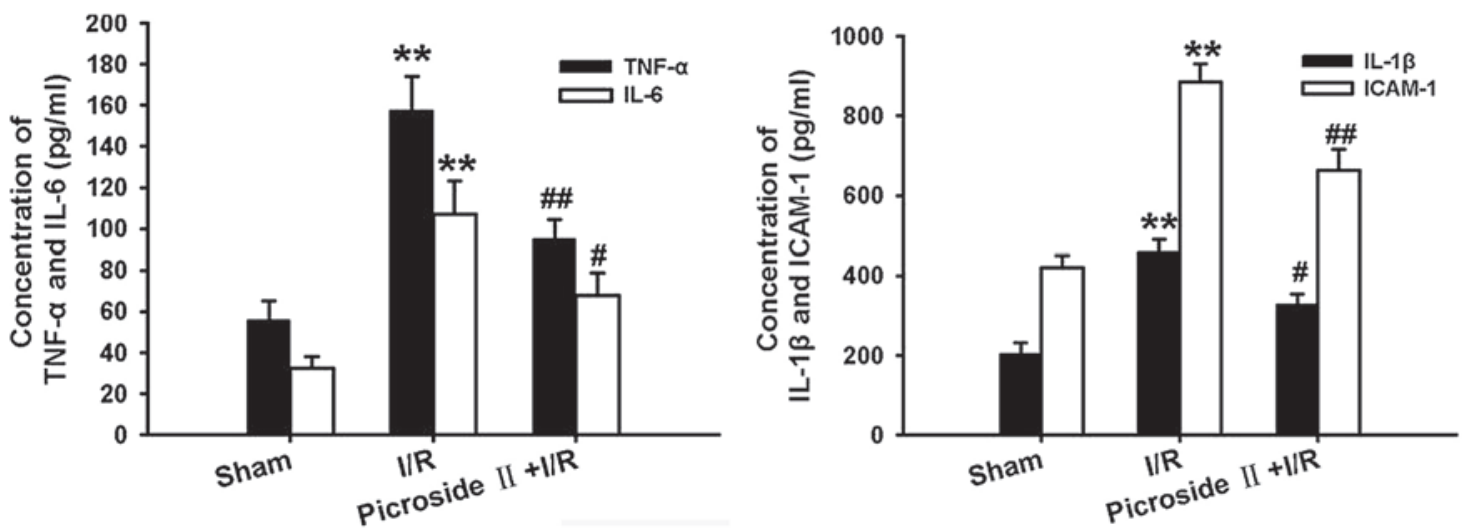

Figure 3. Effect of picroside II on I/R-induced inflammatory factor production in rats ( $\mathrm{n}=8$ in each group). ${ }^{* *} \mathrm{P}<0.01 \mathrm{vs}$. Sham, ${ }^{\#} \mathrm{P}<0.05,{ }^{\# \#} \mathrm{P}<0.01 \mathrm{vs}$. I/R. TNF- $\alpha$, tumor necrosis factor- $\alpha$; IL, interleukin; I/R, ischemia/reperfusion; ICAM-1, intercellular adhesion molecule-1.

emia for $1 \mathrm{~h}$ and reperfusion for $3 \mathrm{~h}$. Consistent with the mRNA expression, the protein expression of RAGE, TLR-2 and TLR-4 was also significantly increased in the I/R group. However, the increased expression of mRNA and protein was remarkably downregulated by pretreatment of picroside II (Fig. 5).

Effect of picroside II on I/R-induced $N F-\kappa B$ activation. Since the activation of NF- $\mathrm{KB}$ is necessary to inflammatory factor production, the NF- $\mathrm{\kappa B}$ activity was investigated in the present study. Following ischemia for $1 \mathrm{~h}$ and reperfusion for $3 \mathrm{~h}$, the NF- $\mathrm{\kappa B}$ activity was increased, as indicated by the increased optical density of NF- $\mathrm{KB}$ p65 in the nuclear lysates. However, this effect induced by $\mathrm{I} / \mathrm{R}$ was significantly inhibited by administration of picroside II (Fig. 6).

\section{Discussion}

In the present study, a protective effect of picroside II on I/R-induced myocardial injury was identified, which was indicated by ameliorating myocardial morphology and decreasing infarct size, cardiomyocyte apoptosis and the activity of CK and $\mathrm{LDH}$. The results also demonstrated that pretreatment with picroside II was able to decrease I/R-induced inflammatory factor production, inhibit HMGB1 expression and release, downregulate RAGE, TLR-2 and TLR-4 expression, and decrease NF- $\kappa$ B activity.

Myocardial I/R injury is a common problem following coronary artery bypass graft, heart transplantation and acute coronary syndrome amongst other problems (22). Although 
A

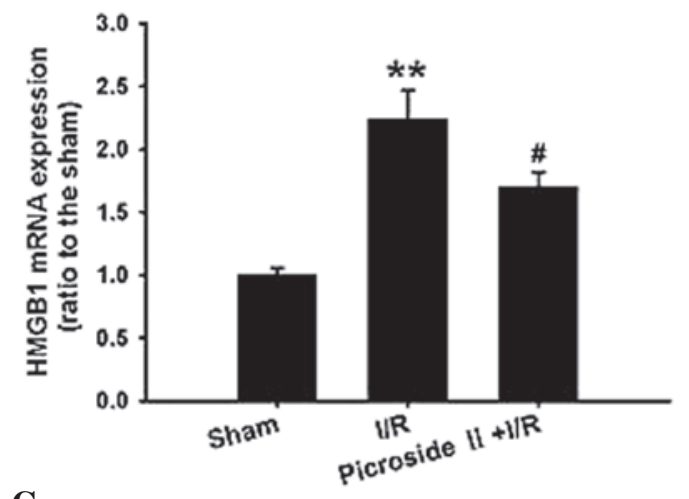

C

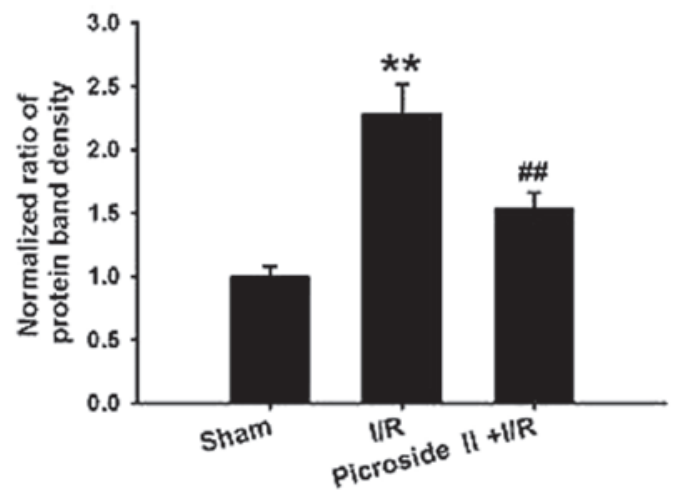

B

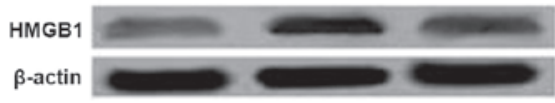

D

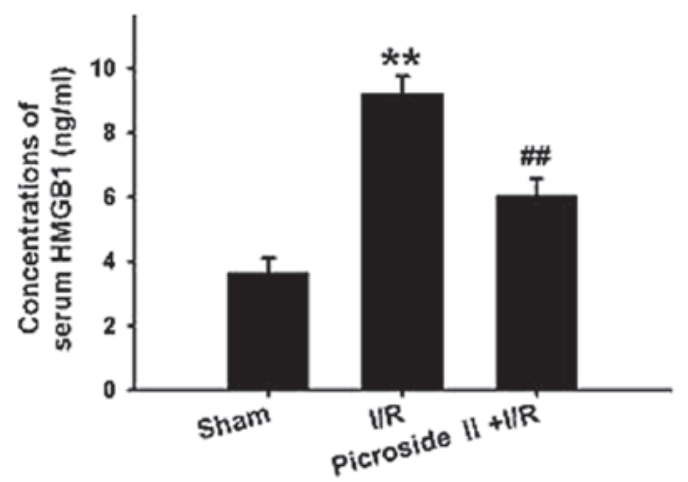

Figure 4. Effect of picroside II on I/R-induced HMGB1 expression and release in rats ( $\mathrm{n}=8$ in each group). (A) HMGB1 mRNA expression by quantitative polymerase chain reaction. (B) Representative image of HMGB1 protein expression by western blotting. (C) Optical density of protein bands. (D) HMGB1 concentration in serum. ${ }^{* *} \mathrm{P}<0.01$ vs. Sham, ${ }^{\# P} \mathrm{P}<0.05$ and ${ }^{\# \#} \mathrm{P}<0.01$ vs. I/R. HMGB1, high mobility group box $1 ; \mathrm{I} / \mathrm{R}$. ischemia/reperfusion.

A

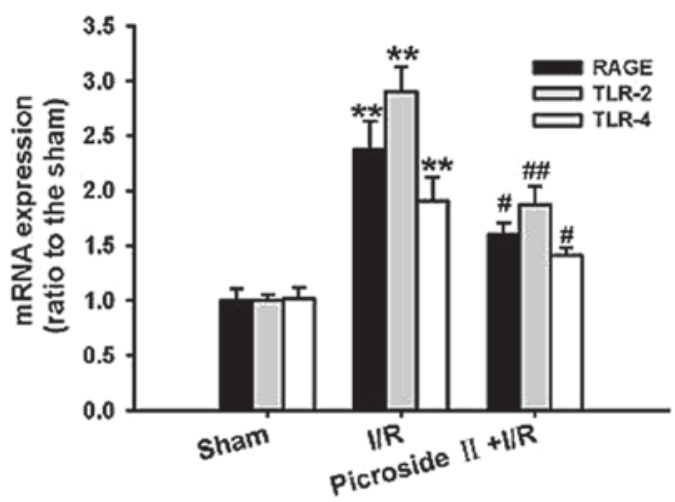

B

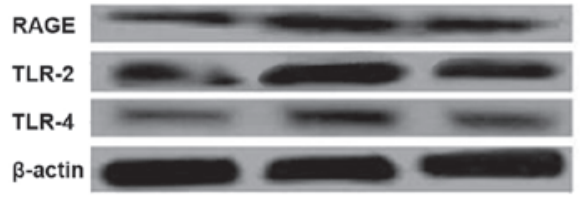

C

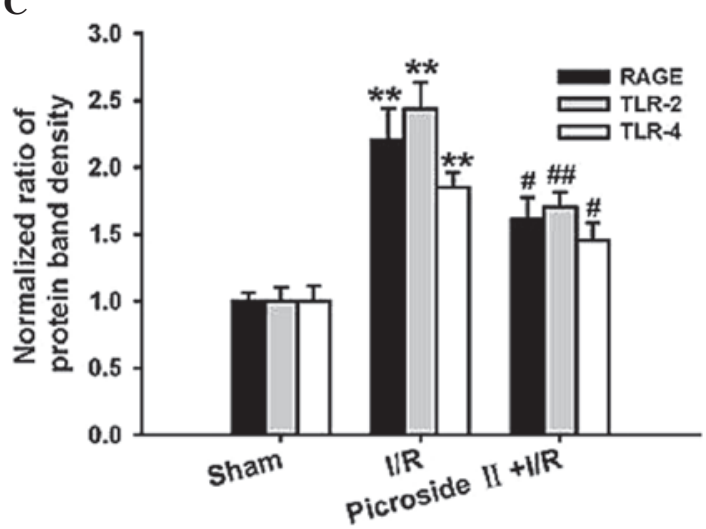

Figure 5. Effect of picroside II on I/R-induced RAGE, TLR-2 and TLR-4 expression in rats (n=8 in each group). (A) mRNA expression by quantitative polymerase chain reaction. (B) Representative image by western Blotting. (C) Optical density of protein bands. ${ }^{* *} \mathrm{P}<0.01$ vs. Sham; ${ }^{\#} \mathrm{P}<0.05$ and ${ }^{\# \#} \mathrm{P}<0.01$ vs. I/R. I/R, ischemia/reperfusion; RAGE, receptor for advanced glycation end products; TLR, toll-like receptor. 


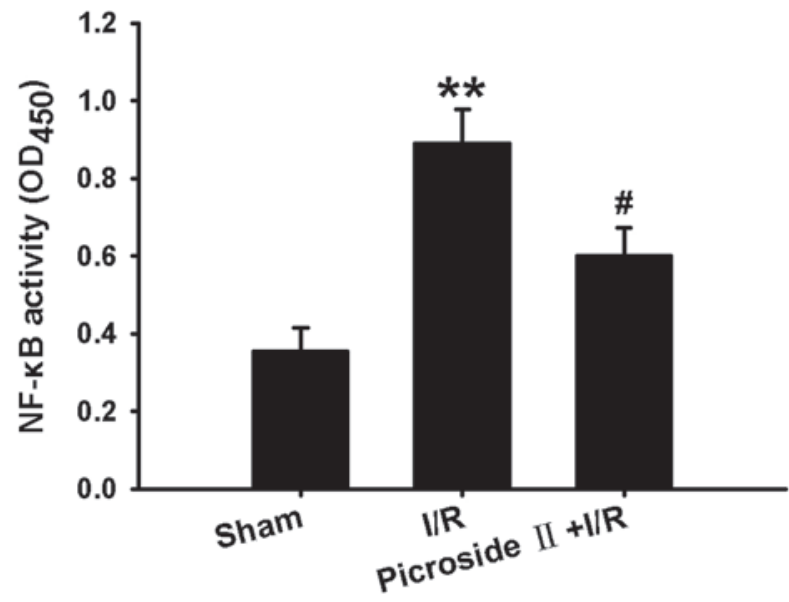

Figure 6. Effect of picroside II on I/R-induced NF- $\kappa \mathrm{B}$ activation in rats ( $n=8$ in each group). Data are expressed as the mean optical density at $450 \mathrm{~nm} \pm$ standard error of the mean. ${ }^{* *} \mathrm{P}<0.01$ vs. Sham; ${ }^{\#} \mathrm{P}<0.05 \mathrm{vs}$. I/R $\mathrm{NF}-\kappa \mathrm{B}$, nuclear factor- $\kappa \mathrm{B} ; \mathrm{I} / \mathrm{R}$, ischemia/reperfusion.

ischemic heart disease is one of the main reasons for morbidity and mortality worldwide, the exact pathophysiological mechanism underlying myocardial I/R injury is not fully understood. Currently, it is well-recognized that the inflammatory response is one of the primary mechanisms underlying myocardial $\mathrm{I} / \mathrm{R}$ injury $(2,3)$. Myocardial I/R may cause local sterile inflammation and produce numerous inflammatory factors, including TNF- $\alpha$, IL-6, IL-1 $\beta$ and ICAM-1 amongst others (23). In addition, myocardial injury is thought to be a result of an intensive inflammatory response initiated by the infiltration of leukocytes and the production of inflammatory factors (22). Infiltrating cells, particularly neutrophils, are directly toxic to the myocardium by releasing proteases and occluding the microvasculature (24). Excessive production of inflammatory factors damages the myocardium not only by triggering deleterious responses but also by amplifying ongoing responses to build a cascade of injury (25). This cascade refers to the excessive production of inflammatory factors could cause vascular endothelial cell damage and increase vascular permeability, and further activate inflammatory cells that increase the inflammatory response (26). Numerous studies have demonstrated that inhibition of the inflammatory response could markedly suppress myocardial injury induced by $I / R$ in various animal models (27-29). Therefore, decrease in inflammation may be a potential therapeutic strategy for myocardial I/R injury.

Recently, numerous studies suggested that the inflammatory response during myocardial $\mathrm{I} / \mathrm{R}$ injury is closely associated with HMGB1 $(6,7)$. HMGB1 is a single peptide chain consisting of 215 amino acid residues with a molecular weight of $\sim 30 \mathrm{kDa}$ (30). It is an evolutionarily conserved nuclear protein in all mammals and is expressed in virtually all types of cells (31). Besides exerting its function in the nucleus, extracellular HMGB1 can mediate various physical or pathological processes, including stimulating inflammatory factor expression and release mainly by binding to its specific receptors, such as RAGE, TLR-2 and TLR-4 (32). Therefore, HMGB1 has been recognized as an important pro-inflammatory mediator and is important in initiating and amplifying the inflammatory response (9-12). In addition, it has been reported that the HMGB1-triggered inflammatory response is crucial in myocardial I/R injury since a decrease in HMGB1 expression and release could significantly inhibit the inflammatory response and ameliorate I/R-induced myocardial injury $(11,33)$. Therefore, inhibition of the HMGB1-mediated inflammatory signaling pathway may be a promising therapeutic strategy for myocardial I/R injury.

Picroside II is an iridoid glucoside isolated from Picrorhiza scrophulariflora Pennell, which has been reported to possess a wide range of pharmacological properties, including anti-oxidative, anti-apoptotic and liver protective effects amongst others (34-36). Furthermore, it has been reported that picroside II is capable of protecting the heart or kidney from I/R-induced injury by inhibition of the inflammatory response $(15,16)$. These studies suggested that picroside II has an anti-inflammation property. Furthermore, studies from our laboratory (Central South University, Changsha, China) and others have demonstrated that picroside II has a protective effect on H/R- or I/R-induced cardiomyocyte injury $(17,18)$, however, the exact mechanism remains unclear. Due to the important role of the inflammatory response in the pathogenesis of myocardial I/R injury and the anti-inflammatory property of picroside II, we hypothesize that the protective mechanism of picroside II on myocardial I/R injury may be associated with the inhibition of the inflammatory response. In the present study, pretreatment with picroside II was observed to inhibit I/R-induced myocardial injury concomitantly by decreasing the inflammatory factor production. As mentioned above, the HMGB1-RAGE/TLR-2/TLR-4-NF- $\kappa$ B signaling pathway is important and the HMGB1-triggered inflammatory response is crucial in myocardial $I / R$ injury. The present study presumes that the anti-inflammatory effect of picroside II may be associated with the suppression of the HMGB1-RAGE/TLR-2/TLR-4-NF- $\kappa$ B signaling pathway. A further study demonstrated that picroside II significantly inhibited I/R-induced inflammatory factor production concomitantly with a decreased HMGB1 expression and release, downregulated expression of RAGE, TLR-2 and TLR-4, and decreased NF- $\kappa$ B activity. These results were concordant with our previously mentioned hypothesis.

In summary, the present study suggested that pretreatment with picroside II was able to protect the myocardium from I/R-induced injury. This beneficial effect was associated, at least partly, with inhibition of the inflammatory response by suppressing the HMGB1-RAGE/TLR-2/TLR-4-NF- $\kappa$ B signaling pathway. The present study provides a theoretical basis regarding the protective effect of picroside II on myocardial ischemia/reperfusion injury, and also serves as a foundation for the development of novel therapies to be used in cases of ischemic heart disease.

\section{Acknowledgements}

This study was supported by the National Nature Science Foundation of China (grant nos. 81460613, 81101476 and 81260337), the Guangxi Nature Science Foundation of China (grantnos.2013GXNSFBA019126and2013GXNSFBA019188), the Bureau of Public Health of Guangxi Province (grant 
no. Z2013206) and the Guangxi Administration of Traditional Chinese Medicine (grant no. GZZC14-36).

\section{References}

1. Eltzschig HK and Eckle T: Ischemia and reperfusion-from mechanism to translation. Nat Med 17: 1391-1401, 2011.

2. Vilahur $\mathrm{G}$ and Badimon L: Ischemia/reperfusion activates myocardial innate immune response: The key role of the toll-like receptor. Front Physiol 5: 496, 2014.

3. Kitano K, Usui S, Ootsuji H, Takashima S, Kobayashi D, Murai H, Furusho H, Nomura A, Kaneko S and Takamura M: Rho-kinase activation in leukocytes plays a pivotal role in myocardial ischemia/reperfusion injury. PLoS One 9: e92242, 2014.

4. Guo J, Wang SB, Yuan TY, Wu YJ, Yan Y, Li L, Xu XN, Gong LL, Qin HL, Fang LH and Du GH: Coptisine protects rat heart against myocardial ischemia/reperfusion injury by suppressing myocardial apoptosis and inflammation. Atherosclerosis 231: 384-391, 2013.

5. Wang Y, Sun J, Liu C and Fang C: Protective effects of crocetin pretreatment on myocardial injury in an ischemia/reperfusion rat model. Eur J Pharmacol 741: 290-296, 2014.

6. Diao H, Kang Z, Han F and Jiang W: Astilbin protects diabetic rat heart against ischemia-reperfusion injury via blockade of HMGB1-dependent NF- $\kappa$ B signaling pathway. Food Chem Toxicol 63: 104-110, 2014.

7. Ding HS, Yang J, Chen P, Yang J, Bo SQ, Ding JW and Yu QQ: The HMGB1-TLR4 axis contributes to myocardial ischemia/reperfusion injury via regulation of cardiomyocyte apoptosis. Gene 527: 389-393, 2013.

8. Hu G,Zhang Y, Jiang H and Hu X: Exendin-4 attenuates myocardial ischemia and reperfusion injury by inhibiting high mobility group box 1 proteinexpression. Cardiol J 20: 600-604, 2013.

9. Tsung A, Tohme S and Billiar TR: High-mobility group box-1 in sterile inflammation. J Intern Med 276: 425-543, 2014.

10. Magna M and Pisetsky DS: The role of HMGB1 in the pathogenesis of inflammatory and autoimmune diseases. Mol Med 20: 138-146, 2014.

11. Herzog C, Lorenz A, Gillmann HJ, Chowdhury A, Larmann J, Harendza T, Echtermeyer F, Müller M, Schmitz M, Stypmann J, et al: Thrombomodulin's lectin-like domain reduces myocardial damage by interfering with HMGB1-mediated TLR2 signalling. Cardiovasc Res 101: 400-410, 2014.

12. Ding HS, Yang J, Gong FL, Yang J, Ding JW, Li S and Jiang YR: High mobility group [corrected] box 1 mediates neutrophil recruitment in myocardial ischemia-reperfusion injury through toll like receptor 4-related pathway. Gene 509: 149-153, 2012.

13. Li JZ, Wu JH, Yu SY, Shao QR and Dong XM: Inhibitory effects of paeoniflorin on lysophosphatidylcholine-induced inflammatory factor production in human umbilical vein endothelial cells. Int J Mol Med 31: 493-497, 2013.

14. Nogueira-Machado JA and de Oliveira Volpe CM: HMGB-1 as a target for inflammation controlling. Recent Pat Endocr Metab Immune Drug Discov 6: 201-209, 2012.

15. Guo Y, Xu X, Li Q, Li Z and Du F: Anti-inflammation effects of picroside 2 in cerebral ischemic injury rats. Behav Brain Funct 6: 43, 2010.

16. Wang L, Liu XH, Chen H, Chen ZY, Weng XD, Qiu T and Liu L: Picroside II decreases the development of fibrosis induced by ischemia/reperfusion injury in rats. Ren Fail 36: 1443-1448, 2014

17. Wu N, Li W, Shu W and Jia D: Protective effect of picroside II on myocardial ischemia reperfusion injury in rats. Drug Des Devel Ther 8: 545-554, 2014.

18. Li JZ, Yu SY, Mo D, Tang XN and Shao QR: Picroside II inhibits hypoxia/reoxygenation-induced cardiomyocyte apoptosis by ameliorating mitochondrial function through a mechanism involving a decrease in reactive oxygen species production. Int J Mol Med 35: 446-452, 2015.
19. Li TT, Zhang YS, He L, Li NS, Peng J and Li YJ: Protective effect of phloroglucinol against myocardial ischaemia-reperfusion injury is related to inhibition of myeloperoxidase activity and inflammatory cell infiltration. Clin Exp Pharmacol Physiol 38: 27-33, 2011.

20. Zhao Q, Hu X, Shao L, Wu G, Du J and Xia J: LipoxinA4 attenuates myocardial ischemia reperfusion injury via a mechanism related to downregulation of GRP-78 and caspase-12 in rats. Heart Vessels 29: 667-6s78, 2014.

21. Livak KJ and Schmittgen TD: Analysis of relative gene expression data using real-time quantitative PCR and the 2- $\Delta \Delta \mathrm{Ct}$ method. Methods 25: 402-408, 2001.

22. Hu X, Zhang K, Xu C, Chen Z and Jiang H: Anti-inflammatory effect of sodium butyrate preconditioning during myocardial ischemia/reperfusion. Exp Ther Med 8: 229-232, 2014.

23. Liang Z, Liu LF, Yao TM, Huo Y and Han YL: Cardioprotective effects of Guanxinshutong (GXST) against myocardial ischemia/ reperfusion injury in rats. J Geriatr Cardiol 9: 130-136, 2012.

24. Turer AT and Hill JA: Pathogenesis of myocardial ischemia-reperfusion injury and rationale for therapy. Am J Cardiol 106: 360-368, 2010.

25. Lin Y, Chen L, Li W and Fang J: Role of high-mobility group box-1 in myocardial ischemia/reperfusion injury and the effect of ethyl pyruvate. Exp Ther Med 9: 1537-1541, 2015.

26. Yu L, Li Q, Yu B, Yang Y, Jin Z, Duan W, Zhao G, Zhai M, Liu L, Yi D, et al: Berberine Attenuates Myocardial Ischemia/Reperfusion Injury by Reducing Oxidative Stress and Inflammation Response: Role of Silent Information Regulator 1. Oxid Med Cell Longev 2016: 1689602, 2016.

27. Hu H, Zhai C, Qian G, Gu A, Liu J, Ying F, Xu W, Jin D, Wang $\mathrm{H}, \mathrm{Hu} \mathrm{H}$, et al: Protective effects of tanshinone IIA on myocardial ischemia reperfusion injury by reducing oxidative stress, HMGB1 expression and inflammatory reaction. Pharm Biol 53: 1752-1758,2015.

28. Zhang R, Wugeti N, Sun J, Yan H, Guo Y, Zhang L, Ma M, Guo X, Jiao C, Xu W, et al: Effects of vagus nerve stimulation via cholinergic anti-inflammatory pathway activation on myocardial ischemia/reperfusion injury in canine. Int J Clin Exp Med 7: 2615-2623, 2014.

29. Zhao ZG, Tang ZZ, Zhang WK and Li JG: Protective effects of embelin on myocardial ischemia-reperfusion injury following cardiac arrest in a rabbit model. Inflammation 38: 527-533, 2015.

30. Shen $X$ and Li WQ: High-mobility group box 1 protein and its role in severe acute pancreatitis. World J Gastroenterol 21: 1424-1435, 2015.

31. Wu AH, He L, Long W, Zhou Q, Zhu S, Wang P, Fan S and Wang $\mathrm{H}$ : Novel mechanisms of herbal therapies for inhibiting HMGB1 secretion or action. Evid Based Complement Alternat Med 2015: 456305, 2015.

32. Yang S, Xu L, Yang T and Wang F: High-mobility group box-1 and its role in angiogenesis. J Leukoc Biol 95: 563-574, 2014.

33. Kang ZC, Jiang WL, Xu Y, Zhu HB and Hou J: Cardioprotection with 8-O-acetyl shanzhiside methylester on experimental myocardial ischemia injury. Eur J Pharm Sci 47: 124-130, 2012.

34. Meng FJ, Hou ZW, Li Y, Yang Y and Yu B: The protective effect of picroside II against hypoxia/reoxygenation injury in neonatal rat cardiomyocytes. Pharm Biol 50: 1226-1232, 2012.

35. Wang L, Liu X, Chen H, Chen Z, Weng X, Qiu T and Liu L: Effect of picroside II on apoptosis induced by renal ischemia/reperfusion injury in rats. Exp Ther Med 9: 817-822, 2015.

36. Gao H and Zhou YW: Anti-lipid peroxidation and protection of liver mitochondria against injuries by picroside II. World J Gastroenterol 11: 3671-3674, 2005. 